\title{
Security and Stability Aspects of Multi Objective Dynamic Economic Dispatch with Renewable Energy and HVDC Transmission Lines
}

\author{
Moses Peter Musau \\ Department of Electrical and Information Engineering, The University of Nairobi, Nairobi, Kenya \\ Email:pemosmusa@uonbi.ac.ke
}

How to cite this paper: Musau, M.P. (2018) Security and Stability Aspects of Multi Objective Dynamic Economic Dispatch with Renewable Energy and HVDC Transmission Lines. Journal of Power and Energy Engineering, 6, 165-187. https://doi.org/10.4236/jpee.2018.69013

\section{Received: July 29, 2016}

Accepted: September 27, 2018

Published: September 30, 2018

Copyright $\odot 2018$ by author and Scientific Research Publishing Inc. This work is licensed under the Creative Commons Attribution International License (CC BY 4.0).

http://creativecommons.org/licenses/by/4.0/

\begin{abstract}
Renewable sources of energy are being integrated into the power grids due to their economic and environmental merits as compared with the traditional fossil-fuel-fired power generation. However, their significant penetration demands a thorough research in terms of system reliability, that is, security and stability. In this paper, Security Constrained Multi Objective Dynamic Economic Dispatch (SCMODED) problem considering cubic thermal cubic cost function, wind, solar penetration, cubic transmission power losses and cubic emissions cost function as objectives is first formulated. Both HVDC and HVAC lines are included in their formulation. Various approaches like probabilistic load flow (PLF), scenario based method, participation factors and Harmony Search algorithm etc. are employed in the solution process. Security and stability effects of renewable energy (RE) penetration are investigated and analyzed. The simulated results reveal that RE penetration leads to reduced cost and emissions and increased security concerns. Further, there is increased power system instability and hence increased load shedding so as to help the power system attain steady state stability. Inclusion of HVDC lines facilitates rapid and fast control to increase the transient stability limit by the action of the converter ignition angle (CIA) and converter extinction angle (CEA).
\end{abstract}

\section{Keywords}

Security Constrained Multi Objective Dynamic Economic Dispatch (SCMODED), Renewable Energy (RE), Stability, HVDC Lines

\section{Introduction to Power System Security}

From the security point of view, the operating conditions of a power system can 
be classified as normal, pre-emergency (alert), emergency or extreme emergency (if the disturbance is very severe) and finally post-emergency (restorative) [1].

Normal operating condition: all the system variables are within the normal range and no equipment is being overloaded. The system operates in a secure manner and is able to withstand a contingency without violating any of the constraints. For normal operating conditions, mandatory security standards exist. Commonly, this concerns the observance of the single-contingency $(N-1)$ criterion, which means that in case of emergency tripping of one important element of a power system, it is kept in normal operation, thus preventing the development of a cascading emergency. A power system operator performs non-stop monitoring of security criteria, ensuring the power reserves needed for frequency and voltage control; a definite reserve is provided to secure the allowable power exchange over the transmission lines. This is managed by continuous energy flow monitoring and limitations.

A pre-emergency condition: setting in when during normal running an element of a power system is tripped, which disturbs its operation. In this case, the emergency condition has not yet begun but the security criteria are no longer observed, and an emergency process may start developing at any time if the severity of the operating condition increases. Still, the situation can be normalized by mobilizing the capacities and reserves. This is handled by automatic and manual switching on of reserves, and fast action on power of electrical plant.

Emergency or Extreme Emergency condition: in the cases when under a pre-emergency state of a power system, countermeasures are not applied or prove inefficient, and emergency processes develop, which can be of local or cascade-wise character. Local emergencies are eliminated by protective means that trip the faulty element, its functions being taken over by other elements of the system. A cascading emergency is followed by other events that still more aggravate its development; as a result, a full collapse of the power system can be expected, with cascading outages of power plants, making it necessary to restore their operation. Once the cascading emergency condition has set in, the process is developing rapidly. The personnel are unable to control the situation any more, and a blackout can only be prevented by fast-acting automatic protection means. Control actions, such as load shedding and controlled system separation, are aimed at preventing a widespread blackout. This condition is handled by stability maintenance measures at actions on the power plant and load, liquidation of out-of-step operation by power system splitting, liquidation of frequency emergency.

Post-Emergency/ Restorative Condition: in the post-emergency condition, the control actions are taken to reconnect all the facilities and to restore system load (i.e. measures for power system restoration to normal state).

\section{SCMODED Review}

SCED problem has been addressed in the past using both the deterministic and hybrid methods. It is worthwhile to note that the heuristic methods have not 
been used to solve such a problem. Deterministic approaches include Interior Point Method (IPM) [2], Markov Decision Process (MDP) [3], General Benders Decomposition (GBD [4], Dual Quadratic Programming (QDP) [5] and Linear Programming (LP) [6]. In all these cases, static single objective SCED is considered. The only Heuristic method used in security studies is Particle Swarm Optimization (PSO) [7]. Hybrid method include Stochastic (FS) Algorithms-Fuzzy logic strategy (FLS) incorporated in Evolutionary Programming (EP) and Tabu-Search (TS) algorithms i.e. FLS-EP and FLS-TS in [5] and Bacterial Foraging (BF), PSO and DE (BF-PSO-DE) [6]. In these two cases, single objective dynamic economic dispatch (SODED) with quadratic cost function and three or four constraints has been utilized. In this paper a five-objective security constrained multi objective DED (SCMODED) with RE, Emission and HVAC \& HVDC line losses will be considered for the first time using a more accurate hybrid method.

\section{SCMODED with RE}

\subsection{Effects of RE on Security}

Power market deregulation, RE integration and economic benefits have resulted in more stressed operating conditions and more vulnerable networks. When the transmission system is most heavily loaded, the power flows through some of the lines are approaching their limits. A slight increase in these flows along with a critical event (a credible contingency) might lead to the development of a cascading emergency and a collapse of the power system. As a consequence, the need to identify the operational security level of the transmission system increases.

RES are being integrated into the power grids due to their economic and environmental merits as compared to the traditional fossil-fuel-fired power generation. However, their significant penetration demands a thorough research in terms of system reliability, system cost, and environmental impact. This project develops a model to include wind and solar farms in the economic dispatch problem. The uncertain nature of the wind speed and solar irradiance is represented by the Weibull pdf. In addition to the classic economic dispatch factors, also included are factors to account for both overestimation and underestimation of available wind and solar power as well as risk factors for the stochastic nature of RE sources. The optimization problem is then numerically solved for a scenario involving two conventional, two wind and one solar powered generators.

\subsection{Effects of RE on Stability}

Power system security is associated with the response of the system to whatever perturbations it is subject to. Normally, security evaluation requires the analysis of dynamic, transient, or voltage stability in the power system. In this paper, we consider Voltage, Frequency and Rotor angle stability. From the discussion in the previous section, it's clear RE penetration reduces stability and thus, makes 
the system insecure. In this case we consider the technical background behind this.

\subsubsection{Voltage Stability}

When power is supplied to a load through a transmission line keeping the sending end voltage, $V_{S}$, the receiving end (load) voltage, $V_{R}$, undergoes variations depending on the magnitude and power factor of the load.

Consider a power system with $P$ and $Q$ as the real and reactive power to be transferred. Further let $R+j X$ be the impedance of the transmission line. From the phasor diagram of the system, then

$$
V_{S}=V_{R}+\frac{P R}{V_{R}}+\frac{Q X}{V_{R}}
$$

The drop in line voltage is thus given by

$$
\Delta V=\frac{P R}{V_{R}}+\frac{Q X}{V_{R}}
$$

But $R \ll X$, then

$$
\Delta V=\frac{Q X}{V_{R}}
$$

Hence the line drop in voltage mainly depends on the flow of reactive power.

The higher the load with small power factor, the greater the voltage variation. The voltage variation at the load is an indication of unbalance between the reactive power generated $Q_{G}$ and that absorbed $Q_{L}$ by the load. When $Q_{G}>Q_{L}$, then the voltage goes up and vice versa. Thus, to keep $V_{R}$ constant for a given $V_{S}$ then $Q$ has to be varied (locally adjusted) since $X$ and $V_{R}$ are constants. The RE sources in this case generate and absorb real and reactive power simultaneously, hence such a design advantage makes them unique in reactive power compensation which is paramount in voltage stability.

Voltage instability is brought about by the inability of the power system to meet the demand of reactive power. In the case of wind energy, if the wind turbine technology utilizes the induction machines (DFIGs) it may lead to a power system's inability to meet the demand of reactive power this is because induction machine consume reactive power thus leading to voltage instability. While in solar energy, photovoltaic use inverters which are designed to operate at unity power factor hence reactive power is neither produced nor absorbed. Hence there is a need to implement a way of voltage control otherwise it may lead to issues with voltage stability.

\subsubsection{Rotor Angle Stability}

In traditional power systems, the rotor angles of synchronous generators are impacted by the changes in active power flow in the system. In a similar way, the power angle $\delta$ between the two voltages $V_{R}$ and $V_{S}$ is given by

$$
\delta=\frac{P X}{V_{R}}-\frac{Q R}{V_{R}}
$$


But $R \ll X$, then

$$
\delta=\frac{P X}{V_{R}}
$$

Hence the power angle mainly depends on the flow of the real power. When there is a change in active power, the synchronizing generators will respond with an electromagnetic torque that will dampen and minimize the rotor angle deviations thus they have synchronizing power.

$\mathrm{RE}$ technology include wind turbines generator and photovoltaic (PV) are asynchronous machines. When such are integrated to the grid via inverters, they make them the power system lose the ability to maintain synchronism; hence they lack synchronizing power and hence this may bring about rotor angle stability. In this paper, the economic benefits of combined real reactive economic dispatch (CRRED) introduced in [8] will be applied in stability analysis.

\subsubsection{Frequency Stability}

The power system frequency $\left(f_{0}\right)$ at any instant is given by the relation

$$
f_{0}=\frac{2 H * \frac{\mathrm{d} f}{\mathrm{~d} t}}{\Delta P}
$$

where $H$ is the inertia constant given by

$$
H=\frac{1}{2} \frac{J W_{m}^{2}}{S_{B}}
$$

where $J$ is the moment of inertia, $W_{m}^{2}$ is the system angular velocity and $S_{B}$ is the base KVA. Further $\mathrm{d} f / \mathrm{d} t$ is the average rate of change of frequency (ROCOF) and $\Delta P$ is the power imbalance given by

$$
\Delta P=P_{G}-P_{D}
$$

where $P_{G}$ the total power is generated and $P_{D}$ is the total load.

Taking $n$ to represent the normal and a abnormal operating conditions (after disturbance), then

$$
f_{n}=\frac{2 H_{n} * \frac{\mathrm{d} f_{n}}{\mathrm{~d} t}}{\Delta P_{n}}
$$

where $\Delta P_{n}=P_{G n}-P_{D n}$.

And

$$
f_{a}=\frac{2 H_{a} * \frac{\mathrm{d} f_{a}}{\mathrm{~d} t}}{\Delta P_{a}}
$$

where $\Delta P_{a}=P_{G a}-P_{D a}$.

The frequency deviation $(\Delta f)$ after disturbance is thus given by

$$
\Delta f=f_{n}-f_{a}=2\left\{\left(\frac{H_{n} * \frac{\mathrm{d} f_{n}}{\mathrm{~d} t}}{P_{G n}-P_{D n}}\right)-\left(\frac{H_{a} * \frac{\mathrm{d} f_{a}}{\mathrm{~d} t}}{P_{G a}-P_{D a}}\right)\right\}
$$


This equation is the one modeled as an optimization problem for minimization.

$$
\min \Delta f=\min 2\left\{\left(\frac{H_{n} * \frac{\mathrm{d} f}{\mathrm{~d} t}}{P_{G n}-P_{D n}}\right)-\left(\frac{H_{a} * \frac{\mathrm{d} f_{a}}{\mathrm{~d} t}}{P_{G a}-P_{D a}}\right)\right\}
$$

Frequency deviation is minimized subject to loads in each bus that sum up to total amount of load during a disturbance, $P_{D a^{*}}$. At the time of load shedding, all the variables are known except for the summation of loads in each bus which give rise to $P_{D a^{*}}$ This value is solved using the harmony search optimization method to find the optimal load at each bus that should be shed to reduce $P_{D a}$ thereby decreasing $\Delta P_{a}$ hence minimizing $\Delta f$ and restore the system frequency level as it should be for the specific IEEE bus system. Some values of ineria $H$ are as shown as follows:

\begin{tabular}{cc}
\hline System Turbine & $\mathrm{H}(\mathrm{s})$ \\
\hline Steam & $4-9$ \\
Gas & $3-4$ \\
Hydro & $2-4$ \\
Wind & $2-5$ \\
Solar PV & 0 \\
\hline
\end{tabular}

Traditionally, electricity generation is fully dispatchable, that is, it is controllable and involves rotating synchronous generators. Via their stored kinetic energy (KE) they add rotational inertia which is a property of frequency dynamics and stability. Rotational inertia, $\mathrm{H}$, minimizes $\Delta f$ in case of frequency deviations rendering frequency dynamics slower thereby increasing available response time to react to fault events for example, line losses, power plant outages or large scale set point changes of either generation or load units.

Low levels of rotational inertia in a power system caused by inverter connected renewable energy sources for example wind and solar PV units that as such do not provide any inertia have implications on frequency dynamics in that they are faster in power systems with low rotational inertia. This can lead to situation where traditional frequency control schemes become too slow for preventing large frequency deviations and their impeding consequences. Loss of rotational inertia and time variance of inertia can lead to new frequency instability phenomena.

The inertia dictates how large the frequency deviations would be due to a sudden change in the generation and load power balance which plays a significant role in maintaining the stability of a power system stability during a transient scenario. The larger the inertia of a system, the smaller the rate of change in rotor speed in the generator during a power imbalance.

Due to the unpredictable nature of the renewable energy that is solar energy 
and wind energy there may be a mismatch between the generation of power and the demand of power. This causes deviations in the system frequency. In the case of a power deficit, the generation is less than the power demand leading to a reduction of speed and hence the system frequency goes down. While if the generation of power is more than the demand, it will cause an increase in speed and hence an increase in the system frequency thus leading to frequency instability.

In wind energy power generation, when fixed speed induction generators are used it contributes to the inertia of a power system because the stator is directly connected to the grid and thus changes in frequency manifests as a change in speed. These speeds are resisted by the rotating mass leading to rotating energy transfer. While in variable speed wind turbines, its rotational speed is decoupled from the grid frequency by electronic converter. Thus variation in grid frequency does not alter the turbine output power. With high wind penetration there is a risk that the power system inertial effect decreases thus aggravating the frequency of the grid.

In solar power generation, the solar power plant consists of the solar cell and DC to AC converter. Hence they do not possess inertia hence won't release energy to grid when frequency. This leads to frequency instability.

\subsection{Security-Stability Analysis}

Security analysis has three major components, namely: Security monitoring, Security assessment, and control. Security monitoring uses real-time system measurements to determine the operating conditions of the system. It checks whether the system is in normal state or not and tells the control what action to take if it is not in a normal state. Security Assessment is a branch of security analysis determines whether the system is secure or not from the next set of probable contingencies. This is the integral part of economic dispatch. If a system goes into emergency states, it is the duty of security control to execute some actions that will restore the system to its normal operating state. This may involve increasing generation of a power plant to meet a sudden increase in load demand, switching of some plants or even simply readjusting the generated output to ensure that the lines are not overloaded. This is called security control.

Integration of RE into the grid and the use of HVDC lines in the modern power system has greatly affected the three aspect of security in one way or another. Security constrained multi objective dynamic Economic Dispatch (SCMODED) in the RE context is the distribution of active and renewable energy production among the power stations so as to meet the minimization of both fuel cost and pollutant emissions simultaneously while ensuring that power is uninterruptedly supplied to the load. This is the problem formulated and studied in this paper.

Contributions: SCMODED with RE and HVDC lines has been formulated for the first time. Security-Stability merits of HVDC lines investigated. More accurate cubic cost functions have been us for the thermal and emissions func- 
tions. Dynamic reactive power from RE sources applied to model the power system stability concepts. A hybrid approach including probabilistic load flow (PLF), Scenario Based Method (SBM), Improved Genetic Algorithm (IGA), Harmony Search and Participation factors has been used in various stages of the solution process.

\subsection{Formulation}

MODED with RE and HVDC transmission line was formulated in [9]. In this paper, the Security aspects of the same problem are investigated. The security constrained MODED (SCMODED) is formulated as

$$
\min f=\min [W F+(1-W) E]
$$

where $W$ is the weighting factor between the fuel cost and emissions. The fuel cost function is defined as

$$
F=\sum_{t=1}^{T}\left[\sum_{i=1}^{N} F\left(P_{i, t, s}\right)+F\left(O C_{t}\right)\right]+\sum_{j=1}^{W} F\left(P_{j, t, s}\right)+\sum_{k=1}^{S} F\left(P_{k, t, s}\right)+F\left(P_{L, i}\right)
$$

where $T$ is the operation time, $N, W$ and $S$ are the total thermal, wind and solar units respectively and $F\left(P_{i, t, s}\right), F\left(P_{j, t, s}\right)$ and $F\left(P_{k, t, s}\right)$ are the corresponding thermal, wind and wind cost functions at time $t$ and scenario $s$ [9].

It is worth to note that $F\left(O C_{t}\right)$ is the outage cost defined by

$$
F\left(O C_{t}\right)=\sum_{s=0}^{S}\left[b \Delta P_{L, t, s}+c \Delta Q_{L, t, s}\right]
$$

where $\Delta P_{L, t, s}$ and $\Delta Q_{L, t, s}$ are the amounts of real and reactive load-shedding in scene $s$ at time $t, b$ and $c$ are the constants of real and reactive load-shedding costs. Combined real and reactive economic dispatch (CRRED) in [8] is applied. Unlike the operating cost, the outage cost determined based on the risk in each scene.

The RE HVAC \& HVDC transmission loses, emissions cost functions are as formulated in [9] where more accurate cubic cost functions have been utilized. It is assumed that the losses due to the REs are negligible since they are located near the load center. Thus the overall cost for the losses is given by

$$
F\left(P_{L, i}\right)=W^{\prime \prime} F\left(P_{L, A C}\right)+\left(1-W^{\prime \prime}\right) F\left(P_{L, D C}\right)
$$

Integration of HVDC lead to a reduction in real losses (11.85\%) and reactive losses (8.09\%) [10]. Thus, by average, the total losses cost can be given by

$$
F\left(P_{L, i}\right)=\left[0.1 W^{\prime \prime}+0.9\right] F\left(P_{L, A C}\right)
$$

where $W^{\prime \prime}$ is a weighting factor defining the losses in the HVAC and HVDC systems. This number depends on the number of HVDC and HVAC lines in the system

The emissions objective function, $E$, is formulated as

$$
E=\sum_{l=1}^{G} E\left(P_{i, t, s}, P_{j, t, s}, P_{k, t, s}\right) ; G=N+W+S
$$


where $G$ is the total number of generators in the system.

The SCMODED is solved Subject to the following constraints.

1) Power Balance Constraints (PBC)

$$
g_{b}\left(P_{b}, Q_{b}\right)=0
$$

- Real power balance

$$
\sum_{i=1}^{N} P_{i, t, s}+\sum_{j=1}^{W} n_{w, t} P_{j, t, s}+\sum_{k=1}^{S} n_{s, t} P_{k, t, s}+\Delta P_{L, t, s}=\sum_{t=1}^{T} P_{D, t}
$$

where $n_{w, t} \in[0,1]$ and $n_{s, t} \in[0,1]$ are control variables used in adjusting the power output of the wind and solar units respectively, $P_{D, t}$ is the system load at time $t$.

- Reactive Power Balance

$$
\sum_{i=1}^{N} Q_{i, t, s}+\sum_{j=1}^{W} n_{w, t} Q_{j, t, s}+\sum_{k=1}^{S} n_{s, t} Q_{k, t, s}+\Delta Q_{L, t, s}=\sum_{t=1}^{T} Q_{D, t}
$$

2) Generation Units Constraints (GUC)

$$
g_{U}\left(P_{i, t, s}, P_{j, t, s}, P_{k, t, s}\right) \leq 0
$$

- Real power generation constraints

$$
\begin{gathered}
P_{i, t, s}^{\min } \leq P_{i, t, s} \leq P_{i, t, s}^{\max } \\
P_{j, t, s}^{\min } \leq P_{j, t, s} \leq P_{j, t, s}^{\max } \\
P_{k, t, s, m}^{\min } \leq P_{k, t, s} \leq P_{k, t, s, m}^{\max }
\end{gathered}
$$

- Reactive power generation constraints

$$
\begin{aligned}
& Q_{i, t, s}^{\min } \leq Q_{i, t, s} \leq Q_{i, t, s}^{\max } \\
& Q_{j, t, s}^{\min } \leq Q_{j, t, s} \leq Q_{j, t, s}^{\max } \\
& Q_{k, t, s}^{\min } \leq Q_{k, t, s} \leq Q_{k, t, s}^{\max }
\end{aligned}
$$

3) System Security Constraints (SSC)

$$
g_{S}\left(P_{i, t, s}, P_{j, t, s}, P_{k, t, s}, S Y\right) \leq 0
$$

- Load shedding limits

$$
\begin{aligned}
& P_{D, t} \geq \Delta P_{L, t, s} \geq 0 \\
& Q_{D, t} \geq \Delta Q_{L, t, s} \geq 0
\end{aligned}
$$

- Multi Scene power constraints

$$
P_{i, t, s}-P_{i, t, s-1} \geq 0
$$

- Ramping speed of thermal generating units constraints

$$
\begin{aligned}
& P_{i, \text { max down }} \leq P_{i, t, s-1}-P_{i, t-1, s} \leq P_{i, \max u p} \\
& P_{i, \max \text { down }} \leq P_{i, t, s}-P_{i, t-1, s-1} \leq P_{i, \max u p}
\end{aligned}
$$

4) System Stability Constraints (SSC)

$$
\left|g_{s s}(h v d c)\right| \leq 0
$$


- Line Flow Constraint (LFC)

$$
\left|P_{t}\right| \leq P_{t, \max }, t=1,2,3, \cdots, N_{t}
$$

where $N_{t}$ the number of lines and $P_{t}$ is the active power flow in the line $t$

- Converter Tap Ratio Constraint (CTRC)

$$
T_{\min } \leq T \leq T_{\max }
$$

- Converter Ignition Angle Constraint (CIAC): Facilitates fast and reliable control of power flows

$$
\alpha_{\min } \leq \alpha \leq \alpha_{\max }
$$

- Converter extinction Angle Constraint (CEAC): Facilitates rapid control to increase transient stability limit

$$
\gamma_{\min } \leq \gamma \leq \gamma_{\max }
$$

- Current Constraint (HCC)

$$
I_{d c, \min } \leq I_{d c} \leq I_{d c, \max }
$$

- HVDC Voltage Constraint (HVC)

$$
V_{d c, \text { min }} \leq V_{d c} \leq V_{d c, \text { max }}
$$

\section{Proposed Methodologies}

\subsection{Probabilistic Optimal Power Flow (POPF)}

The three objective of load flow analysis are to determine the 1) Static operating state of the power system for given loads 2) Voltage magnitude and angle at all the buses and 3) Line flows and system losses. Load flow methods are classified into two deterministic and probabilistic methods. In this paper, probalistic load flow (PLF) is applied since it utilizes different mathematical approaches such as probabilistic approach, fuzzy sets, interval analysis etc. for taking into account uncertainty in RES. Further, it requires inputs with probability density function (PDF) or cumulative density function (CDF) which are the distribution functions of wind speed and solar radiation intensity [11].

The PDF involves buses such as PQ (negative load), PV (voltage controlled), PX and RX. Numerical and Analytical methods are applied in solving the PLF. The analytical methods analyses a system and its inputs using complex mathematical expressions.The load flow equations are linearized hence inaccurate due to the different approximations. On the other hand, numerical methods involve performing DLF a large number of times with inputs of different combinations of nodal power values. Exact non-linear form of load flow equations can be used using Monte Carlo Simulations. Thus, in this paper Numerical Methods will be used although it is time consuming [11].

AC PLF Model for RES

The PLF model is given by

$$
w=f(x), \Delta x=J_{0}^{-1} \Delta w=S_{0} \Delta w
$$




$$
z=g(x), \Delta z=G_{0} J_{0}^{-1} \Delta w=T_{0} \Delta w
$$

Step 1: Input the system data and wind DFIG and Solar PV data.

Step 2: Run the DLF using Newton Raphson (NR) method, so that the expected values of nodal voltages, line flows, $S_{0}$ and $T_{0}$ are obtained.

Step 3: Compute the cumulants of generation and load according to their PDF.

Step 4: Compute the cumulants of the generated active power, absorbed reactive power, power injections and state variables $(\Delta x$ and $\Delta z)$.

Step 5: Obtain the PDF and CDF of $\Delta x$ and $\Delta z$.

\subsection{Scenario-Based Method (SBM)}

For a multivariate function, $y=F(X)$ where $X$ is a vector containing the uncertain input values, the SBM uncertainty modelling is a method for finding the expected value of $y$. A set of scenarios, $\Omega_{s}$ is generated for describing the probable values of $X$ such that;

$$
y=\sum_{s \in \Omega_{s}} \pi_{s} F\left(X_{s}\right)
$$

where $\pi_{s}$ is the probability of state $s$.

Uncertainties and variability in RE power generation and load profile of the system, emerge into a probabilistic MODED which is formulated in this paper. The total cost of energy is given by

$$
C_{T}=\sum_{S, t} \pi_{s} P P_{s}(t) \lambda_{s}(t)+\sum_{i, t} C_{i}\left(P_{i}(t)\right)
$$

where $C_{T}$ is the total cost paid, $\pi_{s}$ is the probability of scenario $s, P P_{s}(t)$ is the purchased power from reserve pool in time $t$, scenario $s$ and $\lambda_{s}(t)$ is the price of energy purchased from the power reserve in time $t$,scenario $s(\$ / \mathrm{MWh})$ and $C_{i}\left(P_{i}(t)\right)$ is the production cost of the $i^{t h}$ thermal unit in time $t$. The objective function of a rational cost that is to be maximized is defined by

$$
F=\sum_{S, t} \pi_{s} P_{D, s}(t) \lambda_{c}(t)-C_{T}
$$

where $\lambda_{c}(t)$ the price of energy in time $t$, and $P_{D, s}(t)$ is the load demand at time $\mathrm{t}$ and scenario $s$.

\subsection{Solution Process for SCMODED}

Step 1: Get the prediction data for the RE power, load and other known conditions using PLF.

Step 2: Set the range of the RE power fluctuation and the risk constant of the load shedding.

Step 3: Build the SCMODED model.

Step 4: Invoke the power system stability and security checks.

Step 5: Solve the SCMODED using Improved Genetic Algorithm (IGA).

\subsection{Optimal Load Shedding Using Harmony Search Method}

Harmony search optimization algorithm is a random search technique that 
draws inspiration from harmony improvisation in music for example jazz and is used widely in the field of optimization. It mimics the rules of various combining pitches and has two distinguishing operators; Harmony Memory Considering Rate (HMCR) and Pitch Adjusting Rate (PAR) that are used in the algorithm to generate and mutate a solution in order to find the optimal solution [12].

This optimization method has garnered great research success in the field of engineering, control and signal processing. Its use is derived from the fact that as musicians compose harmony, they try various possible combinations of the music pitches stored in memory. This search for a perfect harmony is analogous to the procedure of finding optimal solution to various engineering problems.

Variable selection in Harmony search algorithm follows the three rules which are choosing any value from the Harmony Search Memory (HSM), choosing the adjacent value from HSM and finally choosing random value from the possible value range.

The steps for the HAS include the following:

Step 1: Initialize the problem and algorithm parameters, that is, specify objective function together with the various constraints;

Step 2: Initialize harmony memory;

Step 3: Improvise a new harmony by generating a new vector based harmony depending on considerations such as pitch adjustment and random selection;

Step 4: Update harmony memory;

Step 5: Check the stopping criterion.

\subsection{Power System Stability Using Combined Participation Factors and HVDC}

Voltage stability is directly associated with renewable energy with reactive power deficiency. The gradual changes in power systems can lead to shortage of reactive power leading to a reduction of power system stability. Deterioration of power system voltage magnitude and angle increases as load grows and generation becomes varied and intermittent. There is a decrease in voltage at the bus with increased power flow. Further increase in loading leads to shortage of reactive power and any further increase in active power causes a quick decrease in magnitude of voltage at the buses. Hence we can include a reactive power support on the weakest buses which can immediately provide relief and enhance voltage stability. The disturbance is implemented by increasing the load in the PV buses by $50 \%$.

The HVDC lines provide voltage support through controlled reactive power injection from the RE sources. The hybrid losses internal losses are as formulated in the SCMODED the security advantages of the HVDC lines are as in the stability constraints. The load flow problem is performed using combined participation factors (CPF) using a Newton Raphson Solver for the distributed slack bus model (DSB) [8].

The Solution Algorithm for Solving Stability with RE can be represented by the following steps: 
Step 1: Read system data and formulate the $Y_{\text {bus }}$.

Step 2: Initialize bus voltage, phase angles and set initial $P_{\text {loss }}=0$.

Step 3: Set the iteration counter $k=0$ and convergence criteria.

Step 4: Define the participating buses and combined participating factors.

Step 5: Compute $P_{i}^{(k)}, Q_{i}^{(k)}, P_{\text {stat }}^{(k)}, Q_{\text {stat }}^{(k)}$ for the system buses.

Step 6: Evaluate power mismatches.

Step 7: Evaluate the Jacobian matrix.

Step 8: Evaluate the increments of bus voltage magnitude, voltage angle and HVDC stability parameters and phase angles.

Step 9: This process continues until the power mismatches are less than tolerance.

Step 10: Evaluate the real and reactive and reactive power flow and the losses.

\section{Results and Discussion}

\subsection{Effects of Security Settings in SCDED}

As shown in Table 1, ED and SCED cases are compared in terms of generation, losses, optimal cost and emission levels. It is observed that introducing security constraints to secure the power system increases the total generated power, decreases the real losses, increases the reactive drop and increases the generation cost. Further the emission levels are also decreased. The total power generated increases because a secure system is within the line limits and the losses are decreased. The generation cost however increases because of the cost incurred in ensuring the power system is secure.

\subsection{SCMODED with RE}

Three cases will be considered: Case 1: 3 Thermal units, 1 wind thermal unit, 1 solar unit. Case 2: 3 Thermal units, 3 wind units and Case 3: 3 Thermal units, 3 solar units.

Table 1. Optimal generations for ED AND SCED using IGA, demand = $500 \mathrm{MW}$.

\begin{tabular}{ccc}
\hline Generator No. & ED & SCED \\
\hline $\mathrm{P}_{\mathrm{G} 1}$ & 175.54 & 175.93 \\
$\mathrm{P}_{\mathrm{G} 2}$ & 49.25 & 49.30 \\
$\mathrm{P}_{\mathrm{G} 5}$ & 21.63 & 20.82 \\
$\mathrm{P}_{\mathrm{G} 8}$ & 22.29 & 23.68 \\
$\mathrm{P}_{\mathrm{G} 11}$ & 12.62 & 11.89 \\
$\mathrm{P}_{\mathrm{G} 13}$ & 11.48 & 11.25 \\
Total Generation $(\mathrm{MW})$ & 292.80 & 292.87 \\
Total real power losses $(\mathrm{MW})$ & 9.421 & 9.468 \\
Total reactive drop $(\mathrm{MVAR})$ & 6.098 & 7.098 \\
Total generation costs $(\$ / \mathrm{MWh})$ & 1002.323 & 1503.372 \\
Emission (Ton/h) & 340.564 & 312.345
\end{tabular}


These cases are analyzed at increasing demands of $1000 \mathrm{MW}$ and $1500 \mathrm{MW}$ respectively. We begin with a demand of $1000 \mathrm{MW}$. In Table 2, case 1, SCMODED with RE mix is presented which results in optimal cost and the least emissions. In case 2, wind penetration resulted in the least emissions. Use of solar in SCMODED led to the least cost but the highest emissions. This is as shown in case 3. The results for a demand of $1500 \mathrm{MW}$ for the three cases are as shown in Table 3. Comparing with Table 2 it is clear that optimal cost and losses increase with demand while the emissions tend to decrease with increasing demand. This is because the optimal placement of the unified power flow controller (UPFC) at the weakest bus improves the power generated at this bus to be within the line limits hence minimizing the losses and generation cost, hence improving the security.

The security aspects are investigated by adding security constraints to the MODED with RE. When these constraints are added, the total power generated increases as expected for a secure power system. RE integration is of importance, because integrating $\mathrm{RE}$ reduces the losses and generation cost. RE however has

Table 2. Demand $=1000 \mathrm{MW}$.

\begin{tabular}{cccc}
\hline Generator No. & Case1 & Case 2 & Case 3 \\
\hline $\mathrm{P}_{\mathrm{G} 1}$ & 71.06 & 50.91 & 51.23 \\
$\mathrm{P}_{\mathrm{G} 2}$ & 16.70 & 7.62 & -5.81 \\
$\mathrm{P}_{\mathrm{G} 5}$ & 132.44 & 119.06 & 110.67 \\
$\mathrm{P}_{\mathrm{G} 8}$ & 0.50 & 90.08 & 123.23 \\
$\mathrm{P}_{\mathrm{G} 11}$ & 65.63 & 41.78 & 31.61 \\
$\mathrm{P}_{\mathrm{G} 13}$ & 1.65 & -22.91 & -23.93 \\
Total Generation (MW) & 286.98 & 286.54 & 287.00 \\
Total real power losses (MW) & 3.578 & 3.140 & 3.599 \\
Total Generation cost (\$) & 1445.174 & 1309.841 & 1410.508 \\
Emission (Ton/h) & 200.567 & 209.098 & 256.879 \\
\hline
\end{tabular}

Table 3. Demand = $1500 \mathrm{MW}$.

\begin{tabular}{cccc}
\hline Parameter & Case1 & Case 2 & Case 3 \\
\hline $\mathrm{P}_{\mathrm{G} 1}$ & 54.74 & 51.67 & 51.33 \\
$\mathrm{P}_{\mathrm{G} 2}$ & 17.20 & 3.04 & -2.13 \\
$\mathrm{P}_{\mathrm{G} 5}$ & 135.18 & 100.88 & 123.69 \\
$\mathrm{P}_{\mathrm{G} 8}$ & 0.08 & 96.64 & 70.42 \\
$\mathrm{P}_{\mathrm{G} 11}$ & 75.73 & 55.61 & 58.00 \\
$\mathrm{P}_{\mathrm{G} 13}$ & 3.74 & -22.31 & -15.00 \\
Total Generation (MW) & 286.66 & 286.52 & 286.32 \\
Total real power losses (MW) & 3.259 & 3.122 & 2.921 \\
Total generation costs (\$) & 1536.895 & 1489.705 & 1510.732 \\
Emission (Ton/h) & 190.567 & 199.098 & 246.879 \\
\hline
\end{tabular}


control issues as it's intermittent in nature, resulting in one of the busses being weak with high losses. The UPFC installed in the weak bus to improve the power generated to be within the line limits and hence further reduction in losses and cost.

Hence, RE mix integration (Case 1) and installing the control device i.e. UPFC, results in optimal cost and emissions. It is this case that will be analyzed with respect to SCMODED with changing security levels.

\subsection{Confidence Levels for SCMODED with RE Mix}

To demonstrate the effect of the RE sources on the security setting of a power system two cases have been considered as follows: Case 1: The generation cost with thermal units only. Demand of $1000 \mathrm{MW}$. Case 2: The generation cost with thermal, Wind and Solar Units for power demands of 1500 MW and 2000 MW. During the calculation of minimum generation cost in the second case, the risk threshold $P_{a}$ is set to $0.3,0.5$ and 0.9 for the various demands

The results of the case 1 at a demand of $1000 \mathrm{MW}$ are as shown in Table 4 while those for case 2 are as shown in Table 5 and Table 6 for demands of 1000 MW and $1500 \mathrm{MW}$ respectively. Comparing the total costs of generation for the three scenarios it can be seen that the cost of generation is high with all units being thermal as compared to RE mix integrated for the same demand. This can be attributed to the fact that thermal units have high direct costs since they are fuel-dependent while the RE sources are freely available in nature only operational and supply variability costs being incurred.

For the two demands, wind and solar generation in each system under $P_{a}=0.3$ is the lowest, because under that situation each system requires the highest reliability and security. Therefore, it is essential that a larger proportion of power is contributed by the thermal units whose outputs are predictable unlike the RE sources which happen to be stochastic in nature. Under a threshold setting of $P_{a}=$ 0.9 less thermal power is generated by the thermal units and increased generation by the RE mix employed.

Table 4. Optimal generation for five thermal units for a demand of $1000 \mathrm{MW}$.

\begin{tabular}{cc}
\hline Generator No. & Parameter \\
\hline$P_{1}$ & 127.0665 \\
$P_{2}$ & 37.9675 \\
$P_{3}$ & 44.6850 \\
$P_{4}$ & 30.2956 \\
$P_{5}$ & 29.1272 \\
Total Generation & 269.1417 \\
Total real power losses (MW) & 10.1417 \\
Total generation cost (\$) & 1837.2599 \\
Iterations & 263 \\
Emissions (Ton/h) & 230.567
\end{tabular}


Table 5. Optimal generation for $p_{a}=0.3,0.5,0.9$ for a demand of $1500 \mathrm{MW}$.

\begin{tabular}{cccc}
\hline & \multicolumn{3}{c}{ Confidence level } \\
\cline { 2 - 4 } Generator NO. & $p_{a}=0.3$ & $p_{a}=0.5$ & $p_{a}=0.9$ \\
\hline$P_{1}$ & 181.1019 & 180.5352 & 135.8362 \\
$P_{2}$ & 46.7552 & 46.7258 & 45.3369 \\
$W_{1}$ & 16.1533 & 19.1613 & 19.7218 \\
$W_{2}$ & 10.2025 & 10.6094 & 52.1543 \\
$P_{V_{1}}$ & 10.7827 & 10.9198 & 12.0150 \\
Total Generation (MW) & 267.9956 & 267.9514 & 270.0640 \\
Total real power losses (MW) & 8.9956 & 8.9514 & 11.0648 \\
Total generation cost (\$) & 1831.6972 & 1744.9592 & 1744.2422 \\
Emissions (Ton/h) & 210.456 & 200.890 & 234.678 \\
\hline
\end{tabular}

Table 6. Optimal generations for $p_{a}=0.3,0.5,0.9$ for a demand of $2000 \mathrm{MW}$.

\begin{tabular}{cccc}
\hline \multirow{2}{*}{ Generator NO. } & \multicolumn{3}{c}{ Confidence level } \\
\cline { 2 - 4 } & $p_{a}=0.3$ & $p_{a}=0.5$ & $p_{a}=0.9$ \\
\hline$P_{1}$ & 181.126 & 180.6417 & 151.3922 \\
$P_{2}$ & 46.7552 & 46.7289 & 47.6567 \\
$W_{1}$ & 19.1521 & 19.1582 & 36.2761 \\
$W_{2}$ & 10.1912 & 10.5424 & 14.8402 \\
$P_{V_{1}}$ & 10.7730 & 10.8886 & 19.6219 \\
Total Generation (MW) & 267.9975 & 267.9599 & 269.7871 \\
Total real power losses (MW) & 8.9975 & 8.9599 & 10.7869 \\
Total generation cost (\$) & 1915.3274 & 1906.5187 & 1905.5535 \\
Emissions (Ton/h) & 215.456 & 205.890 & 240.678 \\
\hline
\end{tabular}

As observed from Figure 1, as the threshold level increases, the cost of power generation decreases and the emission levels increases. This is due to the fact that wind and solar penetration increases with increasing risk threshold. A higher threshold implies that the power system's security setting allows a larger penetration of sources with unpredictable natures which in this work is DFIGs and PVs.

Figure 2 shows the convergence characteristics for six unit system. From the figure, the problem converged at approximately the $100^{\text {th }}$ iteration. The simulation results demonstrate that the algorithm used is efficient and the solution is reasonable.

The following conclusions can be reached. Integration of RE sources to a power system reduces the overall cost of power generation. The results also show that the average generation cost and emissions levels of RES are dependent on the tolerance level. If the tolerance level is increased, the RE penetration will be increased, which results in less reliability of the power system, and the average generation cost will be decreased and the emissions decreased. Conversely, if the 


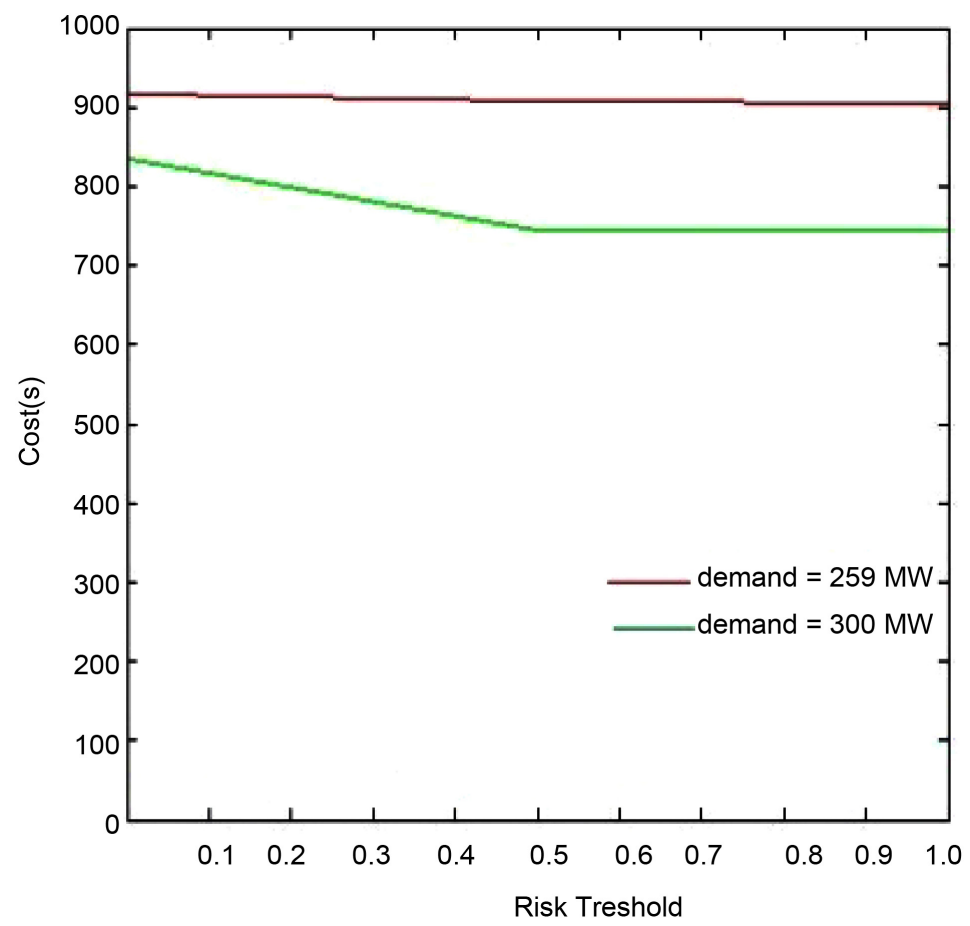

Figure 1. Variation of cost with the risk threshold.

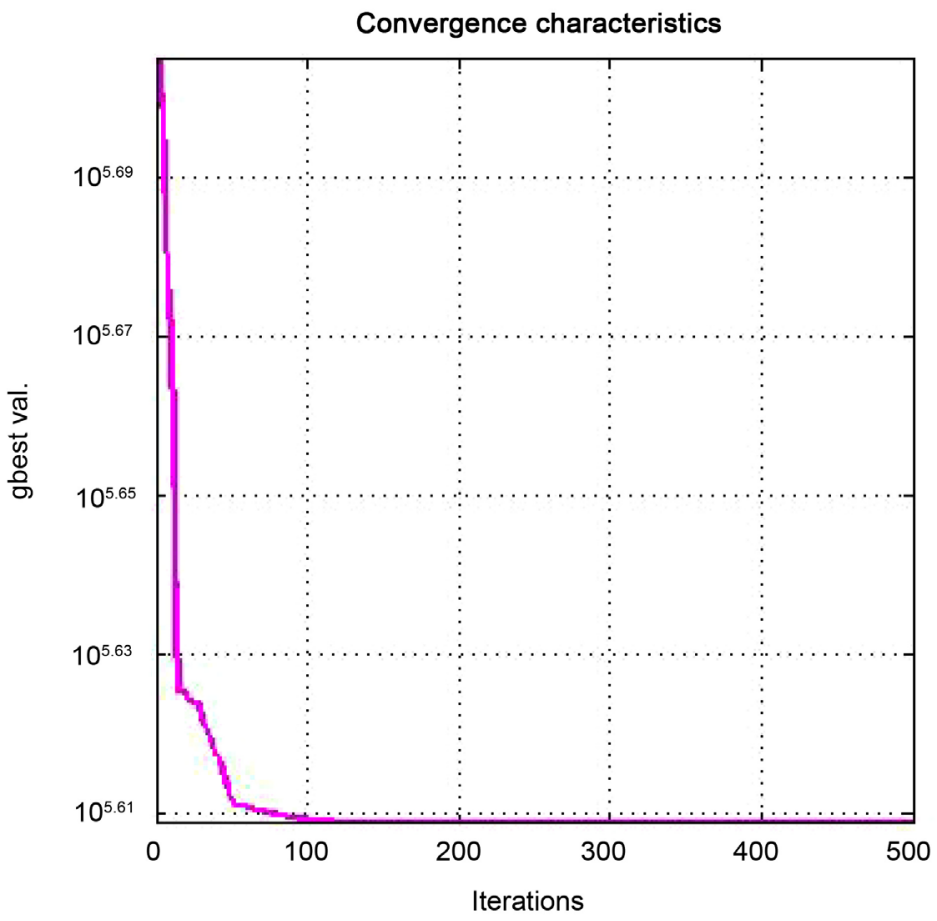

Figure 2. Convergence characteristics for the five-unit system.

tolerance level is decreased, more reliability limits the amount of renewable power to be incorporated in the power system. The average generation cost will thus be increased and the emissions reduced. Scheduling of wind and solar power is not only dependent on their distribution but also on the various associated 
coefficients involved in the cost functions. In addition, since the wind and solar power is stochastic and hence deviates from the scheduled value, an adequate reserve capacity has to be maintained for reliable and stable operation of power system.

\subsection{Stability with RE}

\subsubsection{Voltage Stability: Use of HVDC Lines}

$\mathrm{RE}$ was incorporated in the load flow analysis by including combined participating factors whereby a distributed slack model (DSB) is utilized this is to accommodate the growth of distributed generators such as wind energy and photovoltaic [8]. DSB is based upon distributing the burden of the slack among other generator buses in the power system. To distribute the losses real participation factor was implemented which means the system loss is shared by several generator buses during power flow calculations based on their assigned participation factors. Simulation results are as shown in Table 7 for the injected power, generated power, load and losses.

In normal operating conditions, HVDC lines are introduced, there is a great improvement in the voltage magnitudes thus improving the voltage stability of the power system. This is as shown in Figure 3. In terms of losses, when the HVDC technology is introduced to the RE-incorporated system, the total real and reactive power losses were reduced. This is as shown in Figure 4.

After disturbance, we observe the voltage magnitudes dropped that is as compared to normal operating conditions, the voltage magnitudes reduced. This is well depicted in Figure 5. There is a decrease in voltage at the bus with increased power flow. Further increase in loading leads to shortage of reactive power and any further increase in active power causes a quick decrease in magnitude of voltage at the buses. When the HVDC lines were introduced to the disturbed system, voltage magnitude increased, thus improving the voltage stability after the system is subjected to a disturbance. The losses were also reduced as shown in Figure 6.

Hence, it can be concluded that a HVDC technology is used to control the reactive power which is injected in the power system which greatly improves the

Table 7. Voltage stability for SCMODED using HVDC lines.

\begin{tabular}{ccccccccc}
\hline \multirow{2}{*}{ Case } & \multicolumn{3}{c}{ Injected Power } & \multicolumn{2}{c}{$\begin{array}{c}\text { Generated } \\
\text { Power }\end{array}$} & Load & \multicolumn{2}{c}{ Losses } \\
\cline { 2 - 8 } & MW & MVAR & MW & MW & MVAR & MVAR & MW & MVAR \\
\hline \multicolumn{7}{c}{ Normal Conditions } \\
\hline With HVDC \& HVAC & 2.412 & -33.602 & 191.612 & 73.598 & 189.200 & 107.200 & 2.412 & 9.352 \\
With HVAC & 2.140 & -34.910 & 191.340 & 72.90 & 189.200 & 107.200 & 2.140 & 7.884 \\
\hline \multicolumn{7}{c}{ After Disturbance } \\
\hline With HVDC \& HVAC & 8.611 & -10.613 & 279.961 & 143.137 & 271.350 & 153.750 & 8.611 & 31.768 \\
With HVAC & 7.956 & -15.133 & 279.306 & 138.617 & 271.350 & 153.750 & 7.956 & 27.629 \\
\hline
\end{tabular}




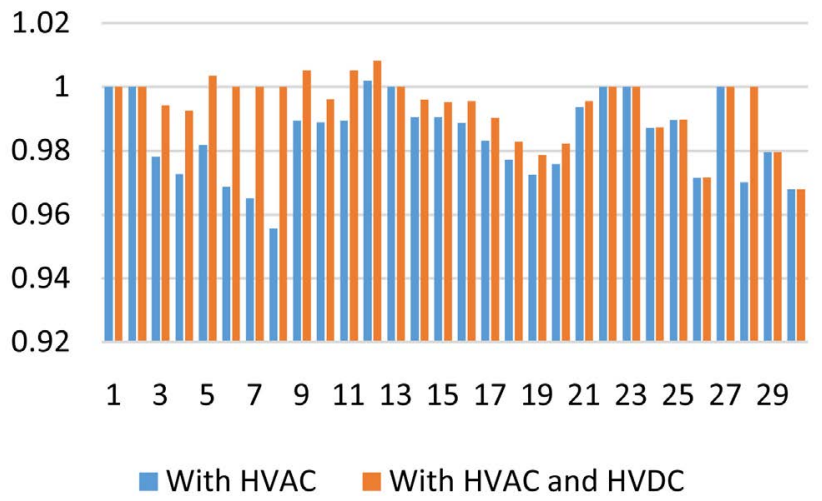

Figure 3. Voltage profile in normal conditions.

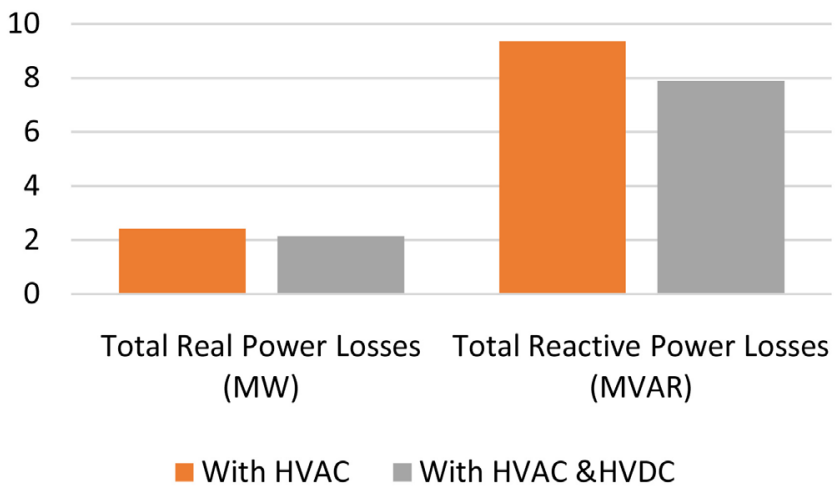

Figure 4. Losses in a normal case.

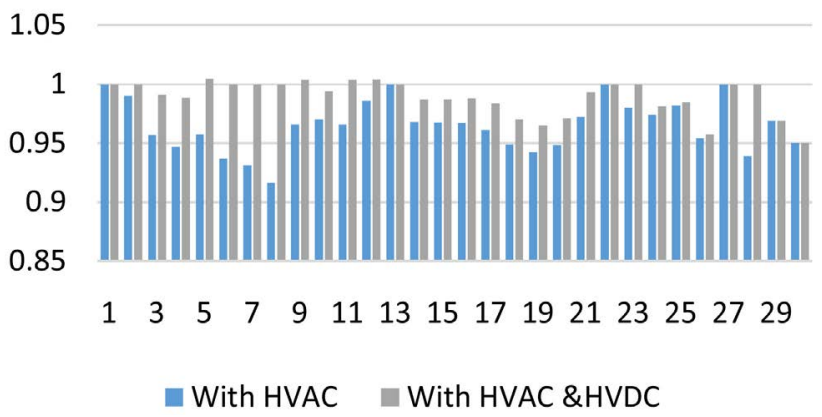

Figure 5. Comparison of voltage profile after disturbance.

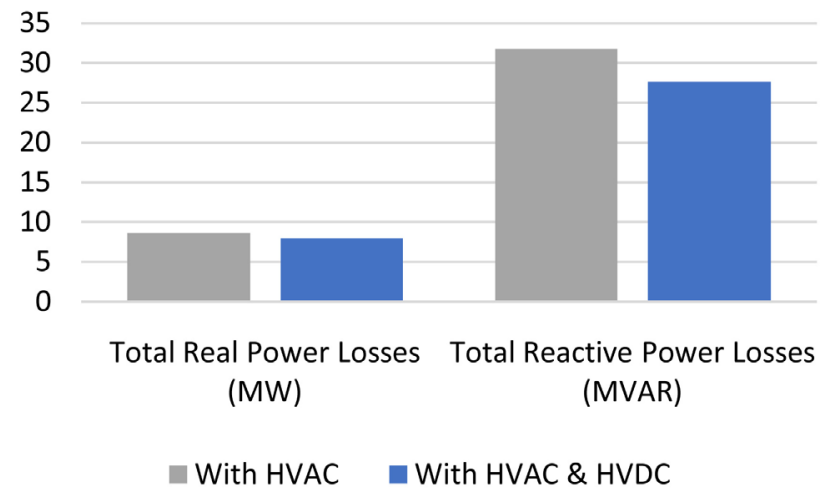

Figure 6. Losses after disturbance. 
voltage stability on the weak buses. This further improves the voltage stability in the whole power system during normal operating conditions and after a disturbance. Furthermore, the real and reactive power losses were also reduced.

\subsubsection{Frequency Stability: Optimal Load Shedding}

The results for optimal load shedding (OLS) for a comparison between CLS and HSLS are as tabulated in Table 8. Comparing the conventional load shedding (CLS) and harmony search load shedding (HSLS), it can be noted that CLS sheds more load which is unnecessary. The harmony search method (HSM) as an optimization tool sheds smaller loads in various buses in finding the optimal combination of loads to shed thereby avoiding unnecessary load shedding. It can also be noted that conventional method (CM) has no regard for load prioritization therefore sheds even the very important loads which is a risky thing.

When RE is introduced in the system, even using HSM as optimization tool (that is, HSLS with RE), there is shedding of more load than in a purely conventional power system. This is as shown in Table 9. Compared to CLS, the power shed in HSM with HVAC Technology is still less despite the fact that there is RE in the system. It can also be observed that when RE is introduced, the amount of reactive energy shed increases drastically.

This can be explained using the concept of Power System Inertia. System inertia is one of the vital system parameters since inertia in rotating masses of synchronous generators and turbines determines immediate frequency response with respect to inequalities in the overall power balance. When a frequency event occurs, synchronous machines either inject or absorb kinetic energy into or from the grid to counteract frequency deviation. The lower the system inertia, the more nervous the grid frequency reacts on abrupt changes in generation and load patterns [13].

$\mathrm{RE}$ systems do not contribute to system reserve and to total system inertia. For instance, solar panels are virtually inertialess since they do not contain any

Table 8. Optimal load shedding for CLS and HSLS.

\begin{tabular}{ccccc}
\hline \multirow{2}{*}{ IEEE System } & \multicolumn{2}{c}{ CLS } & \multicolumn{2}{c}{ HSLS } \\
\cline { 2 - 5 } & MW & MVAR & 40.6136 & MVAR \\
\hline 14 Bus & 66.6 & 13 & 81.3103 & 34.5709 \\
30 Bus & 125 & 31.7 & 269.1929 & 74.2187 \\
57 Bus & 605 & 55.6 & MW \\
\hline
\end{tabular}

Table 9. Optimal load shedding with RE.

\begin{tabular}{ccccc}
\hline \multirow{2}{*}{ IEEE system } & \multicolumn{2}{c}{ HSLS with RE (HVAC) } & \multicolumn{2}{c}{ HSLS with RE (HVDC \& HVAC) } \\
\cline { 2 - 5 } & MW & MVAR & MW & MVAR \\
\hline 14 Bus & 51.2259 & 18.2178 & 49.1345 & 16.9876 \\
30 Bus & 102.0915 & 58.2954 & 97.0097 & 55.1349 \\
57 Bus & 384.2235 & 154.7827 & 374.1156 & 145.9088 \\
\hline
\end{tabular}


moving or rotating parts. Wind turbines have back to back converters which are Doubly Fed Inductive Generators (DFIGs) and Full Converter Synchronous Generator (FCSGs) that electrically decouple the generators from the grid so that no inertial response is delivered during a frequency event although a lot of kinetic energy is stored in the blades and the generators. Wind turbines and solar PV units deliver no inertial response. Adding them to a system or replacing conventional generation by wind and solar generation results in lower system inertia therefore a frequency event leads to very high rate of change of frequency. Increase in rate of change of frequency (ROCOF) can disconnect DGs if they are protected against islanding. In case of major load imbalance, high ROCOF, relays disconnect most of the installed DGs and this disconnection causes further decrease in frequency thereby aggravating the initial contingency.

Renewables are generally exempt from delivering primary or secondary control. Due to their lack of inertia, they increase ROCOF thereby increasing the amount of load shed as seen from the above simulation results. This is especially seen in island grids that already have a lower inertia than the interconnected system. Although renewables without special controls do not contribute to system inertia, stored kinetic energy in wind turbines can support frequency. Solar PV stores no kinetic energy but limited amount of energy in DC capacitor can be controlled together with a battery unit to support frequency control [14]. Inertia constant in wind turbines is 2 to $5 \mathrm{~s}$. Kinetic energy in wind power varies with time. Increasing wind speed increases kinetic energy but for conventional method of power production the value of kinetic energy and thus inertia constant remains constant. When frequency drops, kinetic energy release is proportional to ROCOF and can be controlled independently from ROCOF, theoretically delivering large inertial response than classical power plant. For frequency control, wind turbine or solar PV can be deloaded or curtailed. This is the process where the units operate at sub-optimal operating point creating a power reserve for frequency control. The advantage of this process is that frequency control can be delivered for a longer time. Its downside is that it is relatively expensive due to production support mechanisms and negligible marginal costs. High penetration levels of PV generation can strongly affect power system control and stability from the frequency point of view. They maximize power production therefore there are no power reserves. They also have no rotating parts, therefore no inertial response during major power disturbances. High levels of inertialess PV units reduce the capacity of the system to address frequency deviations during major disturbances affecting power system frequency control [15].

According to Table 9, when the HVDC lines are introduced, the load shedding decreases significantly as compared to the case with HVAC lines only. The use of HVDC lines improves the power system stability in presence of RE in two ways. First, through the action of the converter ignition angle (CIA), which enables fast and reliable control of the real and reactive power flows. Secondly, through the action of the converter extinction angle (CEA), which facilitates 
rapid real and reactive power control to increase the transient stability.

\section{Conclusions}

Integration of RE sources to a power system reduces the overall cost of power generation. Further, the average generation cost and wind and solar penetration are dependent on the tolerance level. If the tolerance level is increased, the wind and solar penetration will be increased, which results in less reliability of the power system, and the average generation cost will be decreased. Conversely, if the tolerance level is decreased, more reliability limits the amount of wind and solar power to be incorporated in the power system. The average generation cost will thus be increased. In short, use of RE reduces cost and emission but makes the system insecure hence unstable.

By introducing HVDC lines under both under normal operating conditions and after a disturbance, the voltage magnitudes were greatly improved, hence improving the voltage stability of the system and it further decreased the real and reactive power losses. From the above discussion, it's seen that introduction of RES in the power system, especially wind and solar that is inertia-less, reduces the capacity of the grid to handle large losses of generation or frequency deviations. Small size and lack of external support of isolated systems could result in severe voltage dips due to disturbances and frequency stability issues. This is the reason why large loads have to be shed in a system with RE during a contingency. This helps the system recover from the high ROCOF faster. Though renewable systems have many advantages, their integration to the grid negatively affects frequency stability of a system and since load shedding is done to restore system frequency, more load is required to be shed for the frequency to return to the nominal value.

Scheduling of wind and solar power is not only dependent on their distribution but also on the various associated coefficients involved in the cost functions. In addition, since the wind and solar power is stochastic and hence deviates from the scheduled value, an adequate reserve capacity has to be maintained for reliable and stable operation of power system. Hence with RE, more load has to be shed so as to help the system attain stability.

\section{Conflicts of Interest}

The author declares no conflicts of interest regarding the publication of this paper.

\section{References}

[1] Hagar, U., Rehtanz, C. and Voropai, N. (2014) Monitoring, Control and Protection of Interconnected Power Systems. Springer, New York. https://doi.org/10.1007/978-3-642-53848-3

[2] Kim, K.-S., Jung, L.-H., Lee, K.Y. and Moon, U.-C. (2006) Security Constrained Economic Dispatch Using Interior Point Method. International Conference on Power System Technology, 12, 1-6. 
[3] Wang, L.Z. and Kong, N. (2010) Security Constrained Economic Dispatch: A Markov Decision Process Approach with Embedded Stochastic Programming. Industrial and Manufacturing Systems Engineering Iowa State University 3016 Black Engineering, Ames, IA, 1-14.

[4] Cvijic, S. and Xiong, J.J. (2011) Security Constrained Unit Commitment and Economic Dispatch through Benders Decomposition: A Comparative Study. Power and Energy Society General Meeting, 10, 1-8.

[5] Granelli, G.P. and Montagna, M. (2000) Security Constrained Economic Dispatch Using Dual Quadratic Programming (QDP). Electric Power Systems Research, 56, 71-80. https://doi.org/10.1016/S0378-7796(00)00097-3

[6] Liu, Y.C., et al. (2015) Computational Study of Security Constrained Economic Dispatch with Multi Stage Resheduling. IEEE Transactions on Power Systems, 30, 920-929.

[7] Gaing, Z.-L. and Chang, R.-F. (2006) Security Constrained Economic Scheduling of Generator Constraints. International Conference on Power System Technology, Chongqing, 22-26 October 2006, 1-7. https://doi.org/10.1109/ICPST.2006.321675

[8] Musau, M.P., Odero, A.N. and Wekesa, C.W. (2015) Combined Real and Reactive Power Economic Dispatch using Multi-Objective Reinforced Learning with Optimized Losses. International Journal of Scientific and Research Publications (IJSRP), 5, No. 10.

[9] Musau, M.P., Odero, A.N. and Wekesa, C.W. (2016) Multi Objective Dynamic Economic Dispatch with Renewable Energy and HVDC Transmission Lines. IEEE PES AFRICA, Livingstone, Zambia, 28 June-2 July 2016, 112-117.

[10] Musau, M.P., Odero, A.N., Wekesa, C.W. and Angela, N.G. (2015) Economic Dispatch for HVDC Bipolar System with HVAC and Optimal Power Flow Comparisons Using Improved Genetic Algorithm (IGA). International Journal of Engineering Research \& Technology (IJERT), 4, 790-799.

[11] https://www.yumpu.com/en/document/view/36393162/power-system-security-anal ysis-with-renewable-energy-iit-mandi

[12] Yang, X.S. (2009) Harmony Search as a Metaheuristic Algorithm, Studies in Computational Intelligence. Vol. 191, Springer, Berlin.

[13] Tielens, P. and Hertem, D.V. (2014) Grid Inertia and Frequency Control in Power Systems with High Penetration of Renewables. Electrical Research Group, Belgium.

[14] Xu, H., Topcu, U., Low, S.H., Clarke, C. and Chandy, K.M. (2005) Load Shedding Probabilities with Hybrid Renewable Power Generation and Energy Storage. California Institute of Technology.

[15] Rahmann, C. and Castillo, A. (2014) Fast Frequency Response Capability of Photovoltaic Power Plants: The Necessity of New Grid Requirement and Definition. University of Chile, Santiago. 